\title{
Probiotic potential of selected lactic acid bacteria strains isolated from Brazilian kefir grains
}

\author{
A. M. O. Leite, ${ }^{*} † \ddagger$ M. A. L. Miguel,§ R. S. Peixoto,§ P. Ruas-Madiedo, $†$ V. M. F. Paschoalin, $\ddagger^{1}$ B. Mayo, $†$ \\ and S. Delgado† \\ *Curso Farmácia, Universidade Federal do Rio de Janeiro, Campus Macaé, Macaé, RJ, Brazil 27930-560 \\ †Instituto de Productos Lácteos de Asturias (IPLA-CSIC), Villaviciosa, Asturias, Spain 33300 \\ łlnstituto de Química, and \\ §Instituto de Microbiologia Paulo de Goes, Universidade Federal do Rio de Janeiro, Rio de Janeiro, RJ, Brazil 21941-904
}

\begin{abstract}
A total of 34 lactic acid bacteria isolates from 4 different Brazilian kefir grains were identified and characterized among a group of 150 isolates, using the ability to tolerate acidic $\mathrm{pH}$ and resistance to bile salts as restrictive criteria for probiotic potential. All isolates were identified by amplified ribosomal DNA restriction analysis and $16 \mathrm{~S}$ rDNA sequencing of representative amplicons. Eighteen isolates belonged to the species Leuconostoc mesenteroides, 11 to Lactococcus lactis (of which 8 belonged to subspecies cremoris and 3 to subspecies lactis), and 5 to Lactobacillus paracasei. To exclude replicates, a molecular typing analysis was performed by combining repetitive extragenic palindromicPCR and random amplification of polymorphic DNA techniques. Considering a threshold of $90 \%$ similarity, 32 different strains were considered. All strains showed some antagonistic activity against 4 model food pathogens. In addition, 3 Lc. lactis strains and $1 \mathrm{Lb}$. paracasei produced bacteriocin-like inhibitory substances against at least 2 indicator organisms. Moreover, 1 Lc. lactis and $2 \mathrm{Lb}$. paracasei presented good total antioxidative activity. None of these strains showed undesirable enzymatic or hemolytic activities, while proving susceptible or intrinsically resistant to a series of clinically relevant antibiotics. The $L b$. paracasei strain MRS59 showed a level of adhesion to human Caco-2 epithelial cells comparable with that observed for Lactobacillus rhamnosus GG. Taken together, these properties allow the MRS59 strain to be considered a promising probiotic candidate. Key words: kefir grain, lactic acid bacteria, lactobacilli, probiotic property
\end{abstract}

Received December 19, 2014.

Accepted February 16, 2015.

${ }^{1}$ Corresponding author: paschv@iq.ufrj.br

\section{INTRODUCTION}

Kefir is a fermented milk product originating from the Northern Caucasus. The name kefir is derived from the Turkish language word keyif, meaning "good feeling" for the feelings experienced after drinking it (Leite et al., 2013b). The fermented beverage is acidic, viscous, and slightly carbonated, and it contains small amounts of alcohol (Leite et al., 2013a). Traditionally, kefir is made by using kefir grains as a starter (Leite et al., 2013a,b). Kefir grains are white to yellowish, cauliflower-like grains, 0.3 to $3.5 \mathrm{~cm}$ in diameter, with a slimy but firm texture. The grains are composed of an inert matrix made up of polysaccharides and proteins. The matrix is densely populated by lactic acid bacteria (LAB) species, acetic acid bacteria, and yeasts (Leite et al., 2012, 2013b).

Kefir beverage has a long tradition of consumption in Eastern Europe, and it is now spreading around the world due to its potential health-associated properties. Although some of the reported health benefits do not yet have well-documented scientific evidence or clinical demonstration, several in vitro and animal studies have associated kefir beverage with alleviation of lactose intolerance (Hertzler and Clancy, 2003), immunomodulation (Hong et al., 2009), antimicrobial activity against pathogenic microorganisms (Chifiriuc et al., 2011), and balance of the intestinal microbiota (Urdaneta et al., 2007). Traditionally, the functional properties of kefir have been attributed mainly to its bioactive peptide content and to kefiran, its main soluble exopolysaccharide (Santos et al., 2003; Rodrigues et al., 2005). However, the potential beneficial effects might also be mediated by the undefined microbial composition of this fermented milk or by the secondary metabolites (Nielsen et al., 2014).

Although a reasonable number of well-characterized probiotic strains are commercially available around the world, screening for novel strains is still of great interest from an industrial point of view (Vinderola et al., 2008; Ayeni et al., 2011). Additionally, strains ex- 
pressing unique and particular characteristics that may enable health benefits may arise in the characterization of natural fermented dairy products such as kefir. This traditional product might be an interesting source of LAB strain with specific functional properties. Even though many authors advocate the importance of human origin as a selective criterion for the search of probiotic strains, an expert panel proposed by FAO/ WHO (2006) suggested that the probiotic activity is more important than the source of the microorganism. In fact, previous reports described the evaluation and selection of kefir LAB isolates for potential use as probiotics (Golowczyc et al., 2008; Zheng et al., 2013).

The aim of the present study was to identify and characterize LAB strains isolated from traditional kefir grains, displaying in vitro properties related to their probiotic potential, according the guidelines recommended by FAO/WHO (2006). After a complete characterization and the corresponding in vivo trials, these strains could be ultimately included as probiotics in functional foods.

\section{MATERIALS AND METHODS}

\section{Isolation of Bacteria from Kefir Grains}

The LAB were isolated by dilution and plating from 4 kefir grains collected in different regions of Brazil. Briefly, $10 \mathrm{~g}$ of each kefir sample was homogenized in $90 \mathrm{~mL}$ of sodium citrate $(2 \%)$. Serial decimal dilutions were obtained and plated on lactobacilli de Man, Rogosa, Sharpe (MRS) and M17 agar media (Difco, Sparks, MD) supplemented with $200 \mu \mathrm{g} / \mathrm{mL}$ of cycloheximide (Sigma-Aldrich, St. Louis, MO), and incubated in aerobic and anaerobic (Gaspak EZ, Difco) conditions at $30^{\circ} \mathrm{C}$ for $72 \mathrm{~h}$. Representative colonies of all morphologies were taken randomly and purified on the same media by subculturing. Gram-positive, catalase-negative isolates were considered as presumptive LAB, which were stored in $15 \%$ glycerol at $-80^{\circ} \mathrm{C}$.

For all subsequent assays, LAB were activated in the corresponding media at $30^{\circ} \mathrm{C}$ for 18 to $24 \mathrm{~h}$, and subcultured in the same conditions.

\section{Tolerance of the Isolates at Low $\mathrm{pH}$}

The ability of the isolates to tolerate low $\mathrm{pH}$ was assayed as described by Nishida et al. (2008) in MRS broth supplemented with $0.2 \%$ sodium thioglycolate (MRS-THIO). In short, overnight cultures were harvested by centrifugation and cells were suspended in PBS (pH 6.5) to obtain an optical density at $600 \mathrm{~nm}$ $\left(\mathbf{O D}_{600}\right)=0.5$. Cell suspensions $\left(\approx 10^{7}\right.$ to $\left.10^{9} \mathrm{cfu} / \mathrm{mL}\right)$ were 10-fold diluted with MRS medium, adjusted to $\mathrm{pH}$
3.0 with $\mathrm{HCl}$, and incubated at $37^{\circ} \mathrm{C}$ for $3 \mathrm{~h}$. The $\mathrm{pH}$ tolerance of the cells was determined by enumerating the viable cells on MRS agar plates. Nontreated cultures used as controls were suspended in conventional, nonacidified MRS ( $\mathrm{pH}$ 6.5).

\section{Bile Tolerance of the Isolates}

Tolerance to bovine bile (Oxgall, Difco) was assayed by growing the isolates in agar plates and broth, following the procedure reported by Delgado et al. (2007) and Guo et al. (2009), respectively. The growth rate of the strains was estimated in MRS-THIO broth in the absence (control) and in the presence (test) of $0.3 \%$ Oxgall. Overnight cultures were inoculated (1\%) into the liquid medium, and cultured at $37^{\circ} \mathrm{C}$ for up to $9 \mathrm{~h}$. Absorbance at $620 \mathrm{~nm}$ was measured every hour. The effect of the bile salts was scored as the time difference required to increase by 0.3 units the absorbance of the culture at $620 \mathrm{~nm}\left(\mathrm{OD}_{620}\right)$ in MRS-THIO broth with and without $0.3 \%$ bile salts. The growth delay (hours) between the culture media was considered as the lag time (LT).

Additionally, tolerance of strains to different concentrations of bile salts was assayed by a plate assay. Individual colonies growing in MRS agar plates were suspended in 2 to $5 \mathrm{~mL}$ of sterile saline solution $0.85 \%$ at a density corresponding to McFarland standard 1. Aliquots of the suspensions $(10 \mu \mathrm{L})$ were spotted onto bile-containing $0.3,0.5,1$, and $2 \%$ (wt/vol) agar plates. The plates were incubated at $37^{\circ} \mathrm{C}$ under anaerobic conditions and growth was recorded after 24 to $48 \mathrm{~h}$. A plate without bile was used as positive control. The experiments were performed in duplicate.

\section{Identification of LAB Isolates}

Total genomic DNA of the isolates was extracted using the GenElute Bacterial Genomic DNA kit (Sigma-Aldrich), following the manufacturer's recommendations. Purified DNA was used as a template to amplify a segment of the $16 \mathrm{~S}$ rRNA gene by the PCR technique using the universal prokaryotic primers SD-Bact-0008-a-S-20 (27F; 5'-AGAGTTTGATCCTGGCTCAG-3') and S-*-Univ-1492R-b-A-21 (1492R; 5'-GGTTACCTTGTTACGACTT-3'). For the amplified ribosomal DNA restriction analysis (ARDRA), amplicons were purified through GenElute PCR CleanUp columns (Sigma-Aldrich), digested with HaeIII and HhaI restriction enzymes (Invitrogen, Paisley, UK) and electrophoresed in agarose gels. Gels were stained with ethidium bromide $(0.5 \mathrm{mg} / \mathrm{mL})$ and photographed under UV light. Representative amplicons of the different ARDRA profiles were sequenced. Sequencing 
was accomplished in an ABI 373 DNA sequencer (Applied Biosystems, Carlsbad, CA). On average, 850 bp of sequence were obtained, which were compared with those deposited in the GenBank database using the BLAST program (http://www.ncbi.nlm.nih.gov/ BLAST/). Following the criterion applied by Palys et al. (1997), sequences with a percentage of identity of $98 \%$ or higher to those in databases were allocated to the same species.

\section{Molecular Typing Analyses}

To exclude replicates, LAB isolates were grouped by both repetitive extragenic palindromic PCR (repPCR), using the primer BOXA2R (5'-ACGTGGTTTGAAGAGATTTTCG-3'), as reported by Koeuth et al. (1995), and random amplification of polymorphic DNA (RAPD) with primer M13 (5'-GAGGGTGGCGGTTCT-3'), as reported by Rossetti and Giraffa (2005). Banding patterns were examined with the Bionumerics 6.5 software program (Applied Maths, Sint-MartensLatem, Belgium) using Dice's coefficient. Cluster analyses of composite data obtained with rep-PCR and RAPD were achieved using the unweighted pair group method with arithmetic averages.

\section{Antimicrobial Activity}

Pathogen Inhibition. The capability of the strains to inhibit a group of foodborne pathogens was determined using an agar spot test as described by Ripamonti et al. (2011). Overnight test cultures were spotted $(2 \mu \mathrm{L})$ on the surface of modified MRS agar (without ammonium citrate and sodium acetate) and incubated anaerobically for $24 \mathrm{~h}$ at $30^{\circ} \mathrm{C}$. Cells were then inactivated with chloroform for $30 \mathrm{~min}$. Escherichia coli ATCC 25922, Salmonella enterica var. Enteritidis ATCC 13076, Staphylococcus aureus ATCC 25923, and Listeria monocytogenes ATCC 15313 were used as indicators. A $100-\mu \mathrm{L}$ volume of an overnight culture of each indicator was mixed with $10 \mathrm{~mL}$ of brain heart infusion (Difco) soft agar (0.7\%), and poured onto MRS agar plates. These were incubated aerobically at $37^{\circ} \mathrm{C}$ for 24 h. Lactobacillus acidophilus ATCC 4356 was used as a negative control. Inhibition but no clear-cut halo or a halo $<1 \mathrm{~mm}$ was recorded as \pm ; a clear zone of growth inhibition around spots $>1 \mathrm{~mm}$ was scored as positive $(+)$; and an inhibition zone between 2 and $5 \mathrm{~mm}$ surrounding the colony was recorded as ++ .

Bacteriocin Production. The production of bacteriocin-like inhibitory substances (BLIS) was successively examined using an agar spot test and a welldiffusion assay. Lactobacillus sakei CECT 906 and Lac- tococcus lactis IL 1403, 2 well-recognized bacteriocinsusceptible strains (Alegría et al., 2010), and Listeria monocytogenes ATCC 15313 were used as indicators. Overnight cultures were spotted on the surface of M17 and MRS agar plates $(0.2 \%$ glucose $)$, incubated for 24 $\mathrm{h}$ at $30^{\circ} \mathrm{C}$ for lactococci and leuconostoc strains, and at $37^{\circ} \mathrm{C}$ for lactobacilli, and inactivated as described previously with chloroform. Spots were covered with 10 $\mathrm{mL}$ of soft agar $(0.75 \%)$ inoculated with the indicators and incubated under the required conditions.

Positive cultures were tested by a well-diffusion assay. Briefly, $20 \mathrm{~mL}$ of agar medium at $45^{\circ} \mathrm{C}$ was vigorously mixed with $200 \mu \mathrm{L}$ of an overnight culture of each indicator and poured into Petri dishes. Supernatants from overnight cultures of the test strains were neutralized to $\mathrm{pH} 6.5$ to 7.0 with $0.1 \mathrm{M} \mathrm{NaOH}$, centrifuged at $10,000 \times g$ for $5 \mathrm{~min}$ at $4^{\circ} \mathrm{C}$, and filter-sterilized through a $0.20-\mu \mathrm{m}$ pore membrane (Millipore, Bedford, MA). Fifty-microliter aliquots of each supernatant were placed in a well excavated in the agar. To allow the diffusion of the supernatant into the agar, plates were maintained at $4^{\circ} \mathrm{C}$ for $1 \mathrm{~h}$ before incubation. Inhibition of the indicators was evaluated after incubation at $37^{\circ} \mathrm{C}$ for $24 \mathrm{~h}$.

To investigate the proteinaceous nature of the BLIS, supernatants were tested in the vicinity of wells filled with $50 \mu \mathrm{L}$ of a solution of either proteinase $\mathrm{K}$ or pronase (each at a concentration of $20 \mathrm{mg} / \mathrm{mL}$ ). After incubation, plates were examined to judge whether the inhibitory substance was sensitive to proteolysis. Lactococcus lactis 1A6, a nisin producer strain (Alegría et al., 2010), was used as control. Tests were performed in duplicate.

\section{Antioxidative Activity}

Sample Preparation for the Antioxidative Assays. Overnight cultures in MRS broth were harvested by centrifugation at $4^{\circ} \mathrm{C}$ for $10 \mathrm{~min}$, washing with isotonic saline solution $(0.85 \%)$ at $4^{\circ} \mathrm{C}$, and suspended in phosphate buffer with $1 \mathrm{~m} M$ EDTA, $\mathrm{pH}$ 7.5. The suspension was adjusted to an $\mathrm{OD}_{600}=1.0$. To obtain the cell extracts, cells were disrupted in a cell disruptor (Constant Systems, Daventry, UK) and deposited immediately in an ice bath. The extracts were then centrifuged at $10,000 \times g$ at $4^{\circ} \mathrm{C}$ for 10 min to eliminate cell debris.

Total Antioxidative Activity. To evaluate the total antioxidative activity (TAA) of the strains, the linolenic acid (LA) test was used as described by Kullisaar et al. (2002), using $45 \mu \mathrm{L}$ of the samples (lysate or whole bacterial cells). The absorbance at $534 \mathrm{~nm}$ was measured on a UV-Vis Spectrophotometer (Hitachi High-Technologies, Tokyo, Japan) and the percentage 
of TAA of the samples was expressed as $\left[1-\left(A_{\mathrm{s}} / A_{\mathrm{c}}\right)\right] \times$ 100], where $A_{\mathrm{s}}$ is the absorbance in the presence of the sample and $A_{\mathrm{c}}$ is the absorbance of the control without sample. Intact cells and cell lysates were assayed in triplicate.

Glutathione Assay. Reduced and oxidized glutathione and the glutathione redox status were evaluated using cell-free extracts and the GSH/GSSG Ratio Assay kit (Millipore, Billerica, MA) following the manufacturer's instructions. The glutathione content was quantified on the basis of a standard curve generated with known amounts of glutathione. The reduced glutathione (GSH) content was calculated as the difference between the total GSH and the oxidized glutathione (GSSG). The glutathione redox ratio was expressed as GSH/GSSG. Escherichia coli CECT 515 was used as a positive control (Masip et al., 2006).

\section{Adhesion Assay}

The epithelial intestinal cell line Caco-2 purchased from the European Collection of Cell Culture (ECACC 86010202) was used to assess the adhesion ability of selected strains. The culture and maintenance of the cell line were carried out following standard procedures (Sánchez et al., 2010) using DMEM medium supplemented with $20 \%$ fetal bovine serum, nonessential aminoacid solution, and a mixture of antibiotics $(50 \mu \mathrm{g} /$ $\mathrm{mL}$ of penicillin-streptomycin, $50 \mu \mathrm{g} / \mathrm{mL}$ of gentamicin, and $1.25 \mu \mathrm{g} / \mathrm{mL}$ of amphotericin B; all reagents from Sigma-Aldrich). The cell line was used after reaching the confluent-differentiated monolayer state $(13 \pm 1 \mathrm{~d})$. The strain Lactobacillus rhamnosus LMG 18243 (also known as $L b$. rhamnosus GG) was used as a reference control for adherence.

Lactobacilli strains, grown overnight in $10 \mathrm{~mL}$ of MRS under standard conditions, were harvested by centrifugation, washed twice in Dulbecco's PBS solution (Sigma-Aldrich), and suspended in supplemented DMEM media without antibiotics, at a concentration of approximately $10^{8} \mathrm{cfu} / \mathrm{mL}$. To remove antibiotics from the cells, monolayers were washed twice in Dulbecco's PBS. Subsequently, the bacterial suspensions were added at a bacteria:eukaryotic cell ratio of 10:1, and incubated for $1 \mathrm{~h}$ at $37^{\circ} \mathrm{C}$ in a $5 \% \mathrm{CO}_{2}$ atmosphere. Afterward, wells were gently washed 3 times with Dulbecco's PBS buffer to remove nonadhered bacteria. Monolayers were disrupted with an EDTA-trypsin solution (Sigma-Aldrich), and the attached bacteria were counted by plating in MRS agar. Adhesion was expressed as the percentage of bacteria adhered with respect to total number of bacteria added. Experiments were carried out using 2 independent Caco- 2 plates $(2$ consecutive passes), and in each plate bacterial strains were analyzed in duplicate.

\section{Safety Assessment}

Hemolysin Production. Hemolysin production was analyzed on Columbia agar plates containing 5\% sheep blood (bioMérieux, Montalieu-Vercieu, France). The presence of $\beta$ - or $\alpha$-hemolysis is indicated by the formation of clear or greenish zones around the colonies, respectively.

Antibiotic Resistance. The MIC of a series of antibiotics was assayed on the selected strains by microdilution in VetMIC plates for LAB (SVA, Uppsala, Sweden), following the manufacturer's recommendations. Colonies grown on LSM (Klare et al., 2005) agar plates were suspended in $5 \mathrm{~mL}$ of sterile saline solution $(0.9 \%)$ to obtain a density corresponding to McFarland standard 1. Suspensions were further diluted 1:1,000 in LSM. One hundred microliters of this dilution was added to each well of the VetMIC plate. The plates were incubated at $37^{\circ} \mathrm{C}$ for $48 \mathrm{~h}$. The MIC were defined as the lowest antibiotic concentration at which no visual growth was observed.

Enzyme Activities. Enzyme activities were measured by the commercial, semiquantitative API-ZYM system (bioMérieux) following the manufacturer's recommendations. In short, $65 \mu \mathrm{L}$ of a cell suspension corresponding to McFarland standard 5 were inoculated in each well of the API-ZYM strips. Enzyme activities were evaluated after $4 \mathrm{~h}$ of incubation in anaerobiosis at $37^{\circ} \mathrm{C}$. Enzyme activities were recorded from 0 (no activity) to 5 ( $\geq 40 \mathrm{nmol}$ of product released) with the API-ZYM color reaction chart.

\section{Statistical Analyses}

Statistical comparisons for both the adhesion and antioxidative tests were performed using the Statistica software package for Windows version 7.0 (Statsoft, Tulsa, OK). Significant differences between treatments were tested by the ANOVA test (1-way ANOVA), followed by a comparison between means using Fisher's least significance difference method, with levels of significance set at $P<0.05$.

\section{RESULTS}

\section{LAB Screening for $\mathrm{pH}$ and Bile-Salt Tolerance}

Thirty-seven out of the 150 tested LAB isolates showed tolerance to $\mathrm{pH} 3.0$ during $3 \mathrm{~h}$ of incubation. However, the growth in these conditions was not com- 
Table 1. Bile resistance of 32 identified and typed lactic acid bacteria isolated from kefir grains

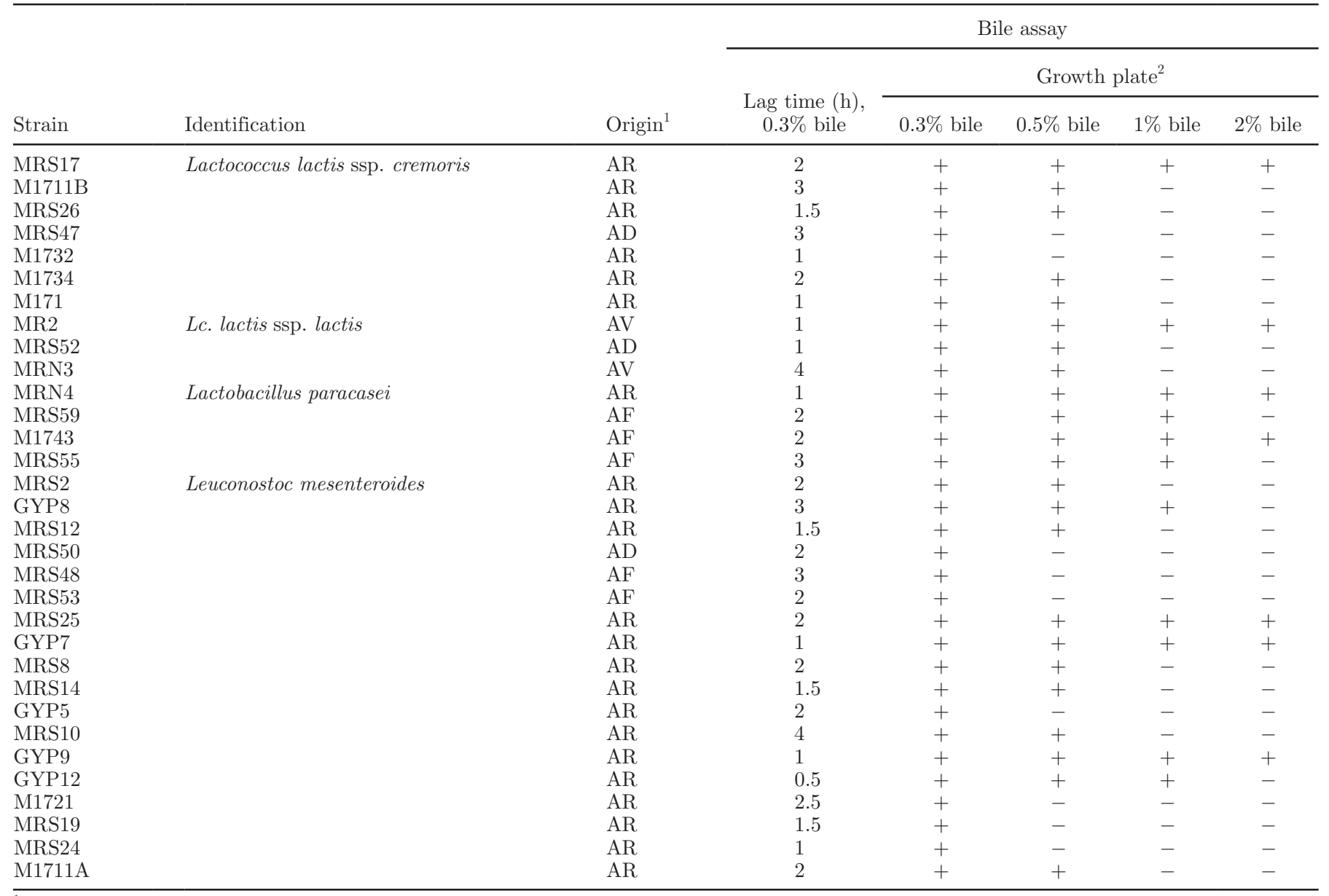

${ }^{1}$ Region of Brazil: $\mathrm{AR}=$ Niterói, RJ; AD = Lavras, MG; AV = Viçosa, RJ; and AF = Alfenas, MG.

${ }^{2}$ Growth plate: $-=$ no growth; $+=$ positive growth.

parable with that in conventional MRS ( $\mathrm{pH} 6.5$ ), with reductions in counts at least 2 logarithmic units compared with controls. These 37 isolates were tested by a plate assay for resistance to different bile concentrations ranging from 0.3 to $2 \%$, and by tolerance in liquid containing $0.3 \%$ of Oxgall. In the latter medium, a LT ranging from 0.5 to $4 \mathrm{~h}$ was observed for 34 isolates (Table 1); these were considered to be bile tolerant. In contrast, $3 \mathrm{LAB}$ isolates presented an LT $>9 \mathrm{~h}$; these were considered bile susceptible.

As a result of the bile plate assay, 34 isolates grew in $0.3 \%, 25$ grew in $0.5 \%, 13$ in $1 \%$, and 8 in $2 \%$ (Table 1 ).

\section{LAB Identification}

Among the 34 isolates 4 different representative ARDRA profiles were observed with the HaeIII and HhaI restriction enzymes (data not shown). Representative $16 \mathrm{~S}$ rDNA amplicons of each of the different profiles were selected for sequencing. Sequence comparisons showed a homology higher than $98 \%$ to 4 different LAB species. Therefore, the 34 isolates were identified as follows: Leuconostoc mesenteroides (18), Lactococcus lactis ssp. cremoris (8), Lactobacillus paracasei (5), and Lactococcus lactis ssp. lactis (3).

\section{LAB Typing}

The LAB isolate typing was performed by a combination of rep-PCR and RAPD techniques. Using a $90 \%$ similarity as threshold (Figure 1), 32 different strains (4 Lb. paracasei, 10 Lc. lactis, and 18 Leu. mesenteroides) were considered. These were all subjected to further analyses.

\section{Antimicrobial Properties}

Antagonistic Activity Against Pathogens. Pathogens were inhibited by most strains in the agar spot test (Table 2). Listeria monocytogenes, E. coli, and 
A

BOXA2R

$\%$ similarity

오.....

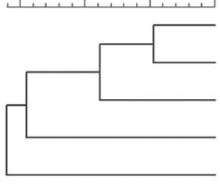

B

$\%$ similarity

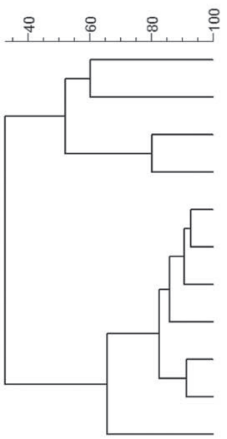

C

\section{$\%$ similarity}

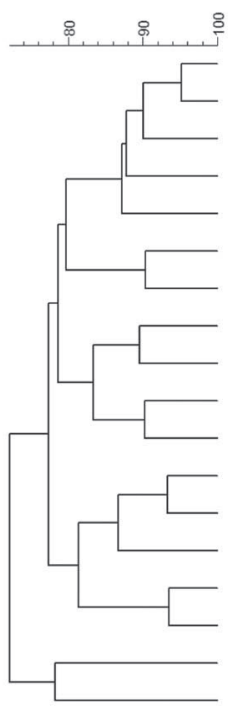

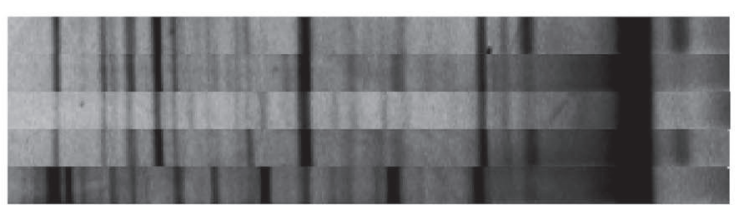

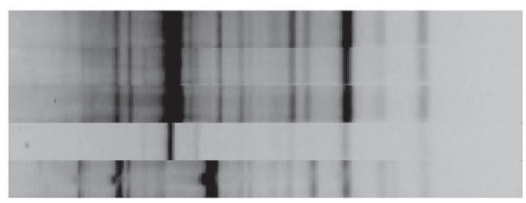

MR1

MRS59

MRN4

M1743

MRS55
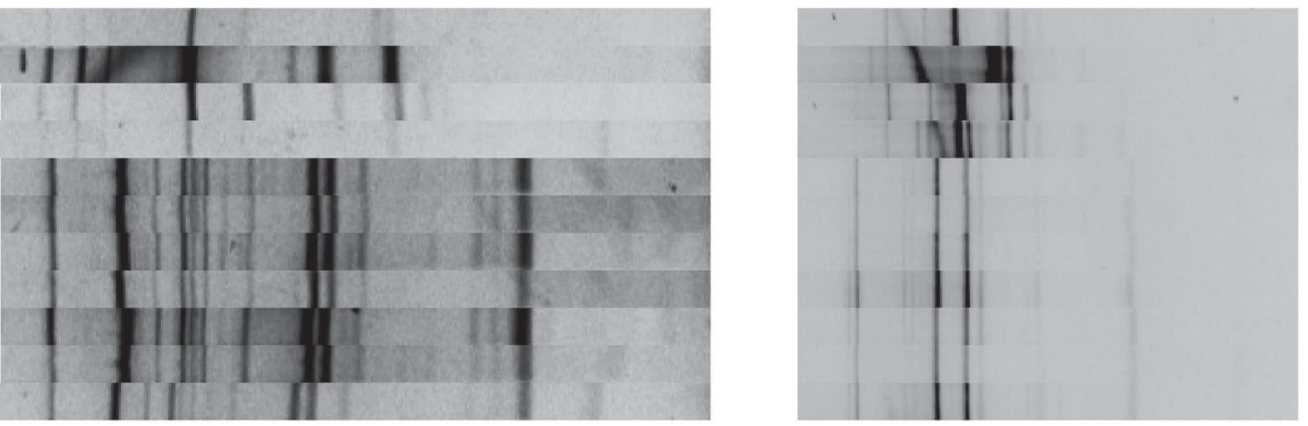

M1711B

MRS52

MR2

MRN3

M1732A

M1734

MRS17

M171

MRS20

MRS26

MRS47

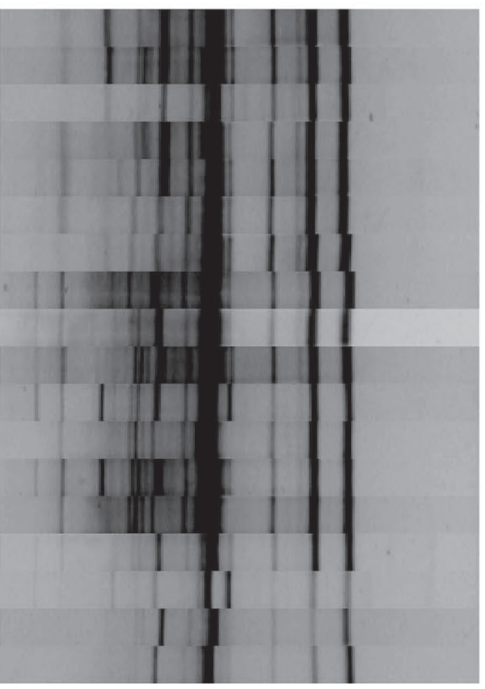

GYP5

M1721

GYP8

MRS25

GYP7

MRS48

MRS53

MRS14

MRS19

M1711A

MRS24

GYP12

MRS50

MRS 10

MRS2

MRS8

GYP9

MRS12

Figure 1. Combined dendrogram obtained from repetitive extragenic palindromic (rep)-PCR and random amplification of polymorphic DNA (RAPD)-PCR profiles, using BOXA2R and M13 primers corresponding to 5 Lactobacillus paracasei strains (panel A), 11 Lactococcus lactis strains (panel B), and 18 Leuconostoc mesenteroides strains (panel C). 
Table 2. Antimicrobial activity data of 32 identified and typed lactic acid bacteria isolated from kefir grains

\begin{tabular}{|c|c|c|c|c|c|c|c|c|c|}
\hline \multirow[b]{2}{*}{ Strain $^{1}$} & \multirow[b]{2}{*}{ Identification } & \multirow[b]{2}{*}{ Origin } & \multicolumn{7}{|c|}{ Inhibition profile spot test ${ }^{2}$} \\
\hline & & & I & II & III & IV & IV & $\mathrm{V}$ & VI \\
\hline MRS17 & Lactococcus lactis ssp. cremoris & $\mathrm{AR}$ & \pm & ++ & - & - & - & - & - \\
\hline M1711B & & $\mathrm{AR}$ & \pm & \pm & - & - & - & - & - \\
\hline MRS26 & & $\mathrm{AR}$ & ++ & - & \pm & ++ & ++ & ++ & ++ \\
\hline M1734 & & AR & - & ++ & - & \pm & \pm & - & \pm \\
\hline M171 & & $\mathrm{AR}$ & ++ & - & \pm & ++ & ++ & ++ & ++ \\
\hline MR2 & Lc. lactis ssp. lactis & AV & ++ & - & - & ++ & ++ & - & - \\
\hline MRS52 & & $\mathrm{AD}$ & - & - & - & ++ & ++ & ++ & ++ \\
\hline MRN3 & & $\mathrm{AV}$ & ++ & \pm & - & ++ & ++ & - & - \\
\hline MRN4 & Lactobacillus paracasei & $\mathrm{AR}$ & \pm & ++ & - & - & - & - & ++ \\
\hline MRS59 & & $\mathrm{AF}$ & ++ & ++ & - & ++ & ++ & - & ++ \\
\hline MRS50 & & $\mathrm{AD}$ & - & - & \pm & \pm & \pm & - & \pm \\
\hline MRS48 & & $\mathrm{AF}$ & - & - & + & \pm & \pm & - & \pm \\
\hline MRS53 & & $\mathrm{AF}$ & - & - & - & - & - & - & - \\
\hline MRS25 & & $\mathrm{AR}$ & \pm & - & - & \pm & \pm & - & \pm \\
\hline GYP7 & & $\mathrm{AR}$ & \pm & - & - & - & - & - & - \\
\hline MRS8 & & AR & - & \pm & - & \pm & \pm & - & - \\
\hline MRS14 & & $\mathrm{AR}$ & - & \pm & - & \pm & \pm & - & - \\
\hline GYP5 & & $\mathrm{AR}$ & + & \pm & - & \pm & \pm & - & - \\
\hline MRS10 & & AR & \pm & \pm & - & \pm & \pm & - & - \\
\hline GYP9 & & $\mathrm{AR}$ & \pm & \pm & - & \pm & \pm & - & - \\
\hline GYP12 & & $\mathrm{AR}$ & - & - & - & - & - & - & - \\
\hline M1721 & & $\mathrm{AR}$ & \pm & \pm & - & \pm & \pm & - & - \\
\hline MRS19 & & $\mathrm{AR}$ & \pm & \pm & - & \pm & \pm & - & - \\
\hline MRS24 & & $\mathrm{AR}$ & \pm & \pm & - & \pm & \pm & - & - \\
\hline
\end{tabular}

${ }^{1}$ Strains in bold produce bacteriocin-like inhibitory substances.

${ }^{2}$ Indicators: I = Escherichia coli ATCC 25922; II = Salmonella enterica serovar Enteritidis ATCC 13076; III = Staphylococcus aureus ATCC 25923; IV = Listeria monocytogenes ATCC 15313; V = Lactobacillus sakei CECT 906; VI = Lactococcus lactis IL1403. Activity: $\pm=$ inhibition but no clear halo; $+=$ presence of a clear zone of growth inhibition around spots $>1 \mathrm{~mm} ;++=$ presence of a clearly defined inhibition zone between 2 and $5 \mathrm{~mm}$ surrounding the colony in the spot test or the wells containing neutralized, cell-free supernatant; - = no inhibition.

S. enterica were inhibited by 23,22 , and 20 strains, respectively. In contrast, $S$. aureus was inhibited by only 7 strains. Pathogen inhibition, however, was not confirmed by the agar well-diffusion assay, with the exception of L. monocytogenes, which was inhibited by 4 strains after neutralization of the cell-free supernatants (see below).

Bacteriocin-Like Inhibitory Substance Production. Lactobacillus sakei CECT 906 was inhibited by 4 strains and Lc. lactis IL 1403 by 12 strains (Table 2 ) in the agar spot assay. The isolates demonstrating antibacterial activity against any of the indicators were subsequently subjected to the well-diffusion assay. None of the Leuconostoc strains showed inhibition against indicators in this assay. Lactococcus lactis IL 1403 was inhibited by 3 lactococci strains (MRS26,
M171, and MRS52). Both L. monocytogenes ATCC 15313 and $L b$. sakei CECT 906 were inhibited by the 3 above-mentioned lactococci strains and one $L b . p a-$ racasei (MRS55) strain. The proteinaceous nature of the BLIS produced by these 4 strains was confirmed by proteinase treatment of the cell-free supernatants.

\section{Antioxidative Activity}

According to Hütt et al. (2006), LAB strains with a TAA value $>20 \%$ are considered to have antioxidative activity. Three of the LAB strains from kefir grains $(2$ Lb. paracasei; MRS 59 and M1743, and 1 lactococcal strain; MRS 52) showed percentages above this value in either intact cells or lysate supernatants (Table 3). All other strains showed mean TAA values below $15 \%$ 
Table 3. Data on total antioxidative activity (TAA) and glutathione determinations (total, reduced, and oxidized glutathione and glutathione redox ratio) of representative lactic acid bacteria strains from kefir grains showing antioxidative activity ${ }^{1}$

\begin{tabular}{|c|c|c|c|c|c|c|c|}
\hline \multirow[b]{2}{*}{ Strain } & \multirow[b]{2}{*}{ Identification } & \multicolumn{2}{|c|}{ TAA test $(\%)$} & \multicolumn{4}{|c|}{ Glutathione test $^{2}(\mu M)$} \\
\hline & & Intact cells & Cell lysate & tGSH & GSSG & GSH & GSH/GSSG \\
\hline MRS52 & $\begin{array}{l}\text { Lactococcus lactis } \\
\text { ssp. lactis }\end{array}$ & $20.3 \pm 3.5^{\mathrm{a}}$ & $11.8 \pm 3.9^{\mathrm{b}}$ & $0.586 \pm 0.23^{\mathrm{b}}$ & $0.062 \pm 0.03^{\mathrm{b}}$ & $0.461 \pm 0.14^{\mathrm{b}}$ & $8.75 \pm 3.19^{\mathrm{a}}$ \\
\hline MRS59 & $\begin{array}{l}\text { Lactobacillus } \\
\text { paracasei }\end{array}$ & $18.4 \pm 4.1^{\mathrm{ac}}$ & $34.1 \pm 9.9^{\mathrm{a}}$ & $0.157 \pm 0.21^{\mathrm{b}}$ & $0.024 \pm 0.01^{\mathrm{b}}$ & $0.110 \pm 0.19^{\mathrm{b}}$ & $5.72 \pm 3.09^{\mathrm{a}}$ \\
\hline MRN4 & Lb. paracasei & $3.5 \pm 2.9^{\mathrm{b}}$ & $14.7 \pm 12.7^{\mathrm{b}}$ & ND & ND & ND & ND \\
\hline $\mathrm{CECT} 15^{3}$ & Escherichia coli & ND & ND & $2.313 \pm 0.48^{\mathrm{a}}$ & $0.31 \pm 0.04^{\mathrm{a}}$ & $1.686 \pm 0.40^{\mathrm{a}}$ & $5.33 \pm 0.67^{\mathrm{a}}$ \\
\hline
\end{tabular}

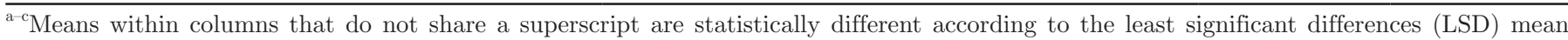
comparison test $(P<0.05)$.

${ }^{1}$ Data are expressed as the means $\pm \mathrm{SD}$, based on 3 replicates.

${ }^{2}$ Glutathione test: $\mathrm{tGSH}=$ total glutathione; GSSG = oxidized glutathione; GSH = reduced glutathione; ND = not determined.

${ }^{3}$ E. coli CECT 515 was used as positive control.

(data not shown), except for 2 other lactobacilli strains (MRS55 and MRN4), which showed a TAA between 15 and $20 \%$ (Table 3 ).

The 3 LAB strains that showed higher values in the TAA test $(P<0.05)$ were further analyzed by the glutathione assay (Table 3 ). The total gluthatione content values found for the LAB strains were lower than the positive control (E. coli; $P<0.05)$. However, the calculated glutathione redox ratio (GSSG/GSH), used to investigate oxidative stress, was similar for all the strains, except for the $L b$. paracasei M1743 strain, in which the oxidized glutathione (GSSG) content was not detectable and, consequently, its GSH/GSSG redox ratio could not be calculated.

Based on the results obtained in the antimicrobial and antioxidative tests, $3 \mathrm{Lb}$. paracasei strains (MRS55, MRS59, and M1743) were considered as presenting probiotic potential and were selected for further analyses.

\section{Adhesion Capability}

The results of the adhesion assay for the 3 selected strains are depicted in Figure 2. Adhesion percentages ranged from 0.9 to $9 \%$. The adhesion level of the MRS59 strain was similar to that of the reference strain GG, whereas those of M1743 and MRS 55 were lower $(P<0.05)$.

\section{Enzymatic Activities and Hemolysin Production}

Undesirable activities, such as trypsin, $\alpha$-chymotrypsin, and $\beta$-glucuronidase activities, were not detected in any of the selected strains. In addition, none of the 3 strains showed hemolytic activity under the assay conditions.

\section{Antibiotic Resistance}

The selected lactobacilli strains were susceptible to all the analyzed antimicrobial agents (including tetracycline, erythromycin, clindamycin, ampicillin, and aminoglycosides) with the exception of vancomycin, for which an intrinsic resistance was observed. The chloramphenicol MIC for MRS55 and M1743 strains was only one dilution higher $(8 \mu \mathrm{g} / \mathrm{mL})$ than the microbiological breakpoint defined by the EFSA $(4 \mu \mathrm{g} / \mathrm{mL})$, which is within the normal acceptable variation around the means (EFSA, 2012).

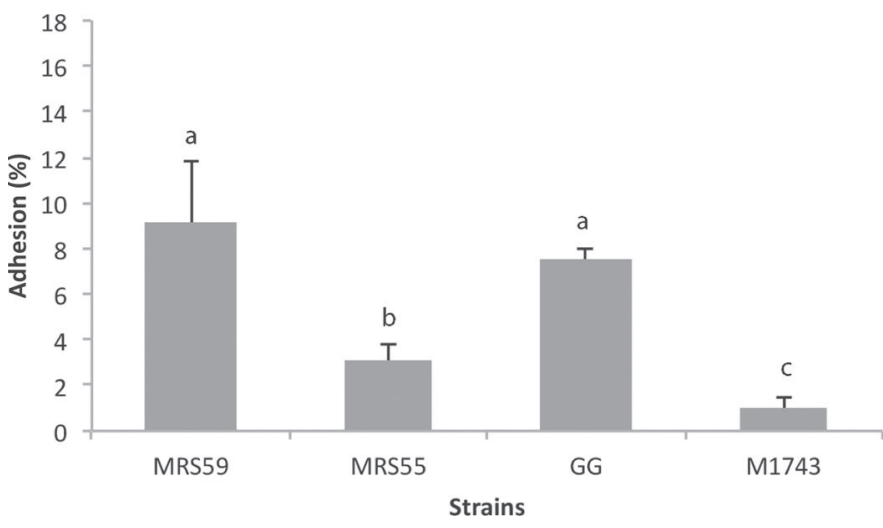

Figure 2. Percentage of adhesion measured as the percentage of colony-forming units per milliliter adhered bacteria with respect to colony-forming units per milliliter of added bacteria of the 3 Lactobacillus paracasei strains to the intestinal epithelial cell line Caco-2. Columns that do not share the same letter are statistically different according to the least significant differences (LSD) mean comparison test $(P<$ 0.05). Lactobacillus rhamnosus GG was used as an adherent reference strain. 


\section{DISCUSSION}

Beyond their technological function, demand is currently increasing for new LAB strain probiotic candidates (Ayeni et al., 2011; Argyri et al., 2013). The complex microbiota of kefir, a traditional beverage endowed with several health benefits (Leite et al., 2013b), could be a source for obtaining novel probiotic strains (Santos et al., 2003; Kumura et al., 2004).

In the present study, LAB were isolated from 4 kefir grain samples, identified and typed by molecular methods, and characterized in vitro for recognized probiosis properties, such as acidity and bile tolerance and antimicrobial and antioxidant activities, key features to consider bacterial strains as probiotics. The secretion of gastric acid and transit through the stomach constitutes a primary defense mechanism that all ingested microorganisms must overcome, including probiotics (Gueimonde and Salminen, 2006). The LAB isolates from kefir grains were screened and selected for their resistance and survival in an acidic environment, as well as for their growth in the presence of $0.3 \%$ bile salts, a similar concentration to that present in the small intestine (Vinderola et al., 2008). Though no scientific consensus exists on the $\mathrm{pH}$ and bile concentration to which probiotic strains should be tolerant (Zago et al., 2011), based on the results, the lactobacilli were able to grow in higher bile concentrations (1\%). Similar results have been previously reported by other authors analyzing LAB strains from different environments (Delgado et al., 2007; Vinderola et al., 2008; Zago et al., 2011; Ramos et al., 2013).

Molecular typing showed a rather high genetic heterogeneity among the LAB isolates from the 4 kefir grains as judged by the large number of different profiles obtained.

Some of these LAB strains exhibited antimicrobial activity against pathogens, and 4 of them produced BLIS, which might provide advantage in competing either in a food product or in the gut (Servin, 2004; Vinderola et al., 2008). Moreover, we observed one Lactococcus BLIS-producing strain, as well as 2 Lb. paracasei, that showed an antioxidative activity higher than those of other tested LAB strains $(P<0.05)$, indicating they may aid in protecting cells from oxidative damage (Lin and Chang, 2000; Zhang et al., 2010). In particular, the lactococci strain showed higher TAA in intact cells; meanwhile, in the $L b$. paracasei strain, the antioxidative activity was more relevant in cell extracts indicating that this activity might be also relevant in case of bacterial lysis into the gastrointestinal tract releasing of the intracellular content. Furthermore, GSH, an important component of the cell defense system against oxidative stress (Masip et al., 2006), was detected for all the LAB strains that showed TAA values $>20 \%$. However, the obtained values suggest that the antioxidative effect displayed by some of these strains could be attained by means of other mechanisms different from glutathione protection, such as enzymatic antioxidants. The antioxidative activities of LAB strains have been under investigation in other studies (Kullisaar et al., 2002; Li et al., 2012; Chen et al., 2014). This protective property may be useful as a defense mechanism in the intestinal microbial ecosystem.

Strains belonging to the Lactobacillus genus are commonly used as probiotics (FAO/WHO, 2006; Gueimonde and Salminen, 2006; Zago et al., 2011). Three $L b$. paracasei strains were considered as appropriate probiotic candidates and selected for further characterization of desirable and undesirable probiotic-related properties.

The capacity to adhere to the intestinal mucosa is an important property for probiotic strains because they should, at least transiently, colonize the host gut (FAO/WHO, 2006). The human colon adenocarcinoma cell line Caco-2 is widely accepted as a model for assessing the adhesion ability of probiotic candidates (Gueimonde and Salminen, 2006; Ayeni et al., 2011). One $L b$. paracasei strain in this study (MRS59) showed similar adhesion values to the recognized probiotic strain $L b$. rhamnosus GG, suggesting it may well be a good in vivo colonizer.

None of these lactobacilli showed $\beta$-glucuronidase activity, which may have negative effects in the colon and has been considered a carcinogenic enzyme (Monteagudo-Mera et al., 2011). In contrast, all 3 strains produced $\beta$-galactosidase, a beneficial enzyme considering both probiotic and technological aspects, supporting the reduction of lactose intolerance as well as milk acidification (Monteagudo-Mera et al., 2011). These strains also presented $\alpha$-glucosidase and $\beta$-glucosidase activity, which could contribute to polysaccharide digestion (Papamanoli et al., 2003). The presence of glycosidase activities in food cultures seems to have an effect on sensory properties because flavor is often linked to sugar metabolism (Papamanoli et al., 2003; Mesas et al., 2011).

In summary, 32 different LAB strains isolated from kefir grains showing good survival under normal gastrointestinal conditions were evaluated in vitro for antimicrobial and antioxidative activities in this study. Among the analyzed strains, $3 \mathrm{Lb}$. paracasei strains were further selected. The presence of harmful enzymatic activities and atypical antibiotic resistances among the selected strains was ruled out. Based on a long history of human consumption, Lb. paracasei, like many other LAB species, is granted a qualified presumption of safety (QPS) status (EFSA, 2011), allowing unrestricted applica- 
tion in food and feed, provided antibiotic resistance is absent. Among the 3 selected strains, Lb. paracasei MRS59 showed the highest number of in vitro probiosis properties; consequently, it was considered as the most appropriate kefir-derived candidate to be used as a probiotic. Further in vivo studies should be also performed to confirm its potential beneficial effects.

\section{ACKNOWLEDGMENTS}

This study was supported by Fundação Carlos Chagas Filho de de Amparo à Pesquisa do Estado do Rio de Janeiro (Rio de Janeiro, Brazil), Coordenação de Aperfeiçoamento de Pessoal de Nível Superior (Brasília, Brazil) Foundation (process number PDEE 5019109), and funds from the Spanish Ministry of Science and Innovation (MICINN, Spain; reference AGL2011-24300 and AGL2012-33278). S. Delgado was supported by a research contract from MICINN under the Juan de la Cierva Program (reference JCI-2008-02391).

\section{REFERENCES}

Alegría, A., S. Delgado, C. Roces, B. Lopez, and B. Mayo. 2010. Bacteriocins produced by wild Lactococcus lactis strains isolated from traditional, starter-free cheeses made of raw milk. Int. J. Food Microbiol. 143:61-66.

Argyri, A. A., G. Zoumpopoulou, K.-A. G. Karatzas, E. Tsakalidou, G.-J. E. Nychas, E. Z. Panagou, and C. C. Tassou. 2013. Selection of potential probiotic lactic acid bacteria from fermented olives by in vitro tests. Food Microbiol. 33:282-291.

Ayeni, F. A., B. Sanchez, B. A. Adeniyi, C. G. de Los Reyes-Gavilan, A. Margolles, and P. Ruas-Madiedo. 2011. Evaluation of the functional potential of Weissella and Lactobacillus isolates obtained from Nigerian traditional fermented foods and cow's intestine. Int. J. Food Microbiol. 147:97-104.

Chen, P., Q. Zhang, H. Dang, X. Liu, F. Tian, J. Zhao, Y. Chen, H. Zhang, and W. Chen. 2014. Screening for potential new probiotic based on probiotic properties and $\alpha$-glucosidase inhibitory activity. Food Contr. 35:65-72.

Chifiriuc, M. C., A. B. Cioaca, and V. Lazar. 2011. In vitro assay of the antimicrobial activity of kephir against bacterial and fungal strains. Anaerobe 17:433-435.

Delgado, S., E. O'Sullivan, G. Fitzgerald, and B. Mayo. 2007. Subtractive screening for probiotic properties of Lactobacillus species from the human gastrointestinal tract in the search for new probiotics. J. Food Sci. 72:M310-M315.

EFSA. 2011. EFSA Panel on Biological Hazards (BIOHAZ). Scientific Opinion on the maintenance of the list of QPS biological agents intentionally added to food and feed (2011 update). EFSA J. 9:82.

EFSA. 2012. EFSA Panel on Additives and Products or Substances used in Animal Feed (FEEDAP): Guidance on the assessment of bacterial susceptibility to antimicrobials of human and veterinary importance. EFSA J. 10:10.

FAO/WHO. 2006. Probiotic in foods. Health and nutritional properties and guidelines for evaluation. Vol. 85. FAO Food and Nutrition Paper, Rome, Italy.

Golowczyc, M. A., M. J. Gugliada, A. Hollmann, L. Delfederico, G. L. Garrote, A. G. Abraham, L. Semorile, and G. De Antoni. 2008. Characterization of homofermentative lactobacilli isolated from kefir grains: Potential use as probiotic. J. Dairy Res. 75:211-217.

Gueimonde, M., and S. Salminen. 2006. New methods for selecting and evaluating probiotics. Dig. Liver Dis. 38:S242-S247.
Guo, Z., J. Wang, L. Yan, W. Chen, X.-m. Liu, and H.-p. Zhang. 2009. In vitro comparison of probiotic properties of Lactobacillus casei Zhang, a potential new probiotic, with selected probiotic strains. Lebenson. Wiss. Technol. 42:1640-1646.

Hertzler, S. R., and S. M. Clancy. 2003. Kefir improves lactose digestion and tolerance in adults with lactose maldigestion. J. Am. Diet. Assoc. 103:582-587.

Hong, W.-S., H.-C. Chen, Y.-P. Chen, and M.-J. Chen. 2009. Effects of kefir supernatant and lactic acid bacteria isolated from kefir grain on cytokine production by macrophage. Int. Dairy J. 19:244-251.

Hütt, P., J. Shchepetova, K. Loivukene, T. Kullisaar, and M. Mikelsaar. 2006. Antagonistic activity of probiotic lactobacilli and bifidobacteria against entero- and uropathogens. J. Appl. Microbiol. 100:1324-1332.

Klare, I., C. Konstabel, S. Muller-Bertling, R. Reissbrodt, G. Huys, M. Vancanneyt, J. Swings, H. Goossens, and W. Witte. 2005. Evaluation of new broth media for microdilution antibiotic susceptibility testing of Lactobacilli, Pediococci, Lactococci, and Bifidobacteria. Appl. Environ. Microbiol. 71:8982-8986.

Koeuth, T., J. Versalovic, and J. R. Lupski. 1995. Differential subsequence conservation of interspersed repetitive Streptococcus pneumoniae BOX elements in diverse bacteria. Genome Res. 5:408418.

Kullisaar, T., M. Zilmer, M. Mikelsaar, T. Vihalemm, H. Annuk, C. Kairane, and A. Kilk. 2002. Two antioxidative lactobacilli strains as promising probiotics. Int. J. Food Microbiol. 72:215-224

Kumura, H., Y. Tanoue, M. Tsukahara, T. Tanaka, and K. Shimazaki. 2004. Screening of dairy yeast strains for probiotic applications. J. Dairy Sci. 87:4050-4056.

Leite, A. M. O., D. C. Leite, E. M. Del Aguila, T. S. Alvares, R. S. Peixoto, M. A. Miguel, J. T. Silva, and V. M. Paschoalin. 2013a. Microbiological and chemical characteristics of Brazilian kefir during fermentation and storage processes. J. Dairy Sci. 96:41494159 .

Leite, A. M. O., B. Mayo, C. T. C. C. Rachid, R. S. Peixoto, J. T. Silva, V. M. F. Paschoalin, and S. Delgado. 2012. Assessment of the microbial diversity of Brazilian kefir grains by PCR-DGGE and pyrosequencing analysis. Food Microbiol. 31:215-221.

Leite, A. M. O., M. A. Miguel, R. S. Peixoto, A. S. Rosado, J. T Silva, and V. M. Paschoalin. 2013b. Microbiological, technological and therapeutic properties of kefir: A natural probiotic beverage. Braz. J. Microbiol. 44:341-349.

Li, S., Y. Zhao, L. Zhang, X. Zhang, L. Huang, D. Li, C. Niu, Z. Yang, and Q. Wang. 2012. Antioxidant activity of Lactobacillus plantarum strains isolated from traditional Chinese fermented foods. Food Chem. 135:1914-1919.

Lin, M. Y., and F. J. Chang. 2000. Antioxidative effect of intestinal bacteria Bifidobacterium longum ATCC 15708 and Lactobacillus acidophilus ATCC 4356. Dig. Dis. Sci. 45:1617-1622.

Masip, L., K. Veeravalli, and G. Georgiou. 2006. The many faces of glutathione in bacteria. Antioxid. Redox Signal. 8:753-762.

Mesas, J. M., M. C. Rodriguez, and M. T. Alegre. 2011. Characterization of lactic acid bacteria from musts and wines of three consecutive vintages of Ribeira Sacra. Lett. Appl. Microbiol. 52:258-268.

Monteagudo-Mera, A., I. Caro, L. B. Rodriguez-Aparicio, J. Rua, M. A. Ferrero, and M. R. Garcia-Armesto. 2011. Characterization of certain bacterial strains for potential use as starter or probiotic cultures in dairy products. J. Food Prot. 74:1379-1386.

Nielsen, B., G. C. Gurakan, and G. Unlu. 2014. Kefir: A multifaceted fermented dairy product. Probiotics Antimicrob. Proteins 6:123-135.

Nishida, S., A. Michinaka, K. Nakashima, H. Iino, and T. Fujii. 2008. Evaluation of the probiotic potential of Lactobacillus paracasei KW3110 based on in vitro tests and oral administration tests in healthy adults. J. Gen. Appl. Microbiol. 54:267-276.

Palys, T., L. K. Nakamura, and F. M. Cohan. 1997. Discovery and classification of ecological diversity in the bacterial world: The role of DNA sequence data. Int. J. Syst. Bacteriol. 47:1145-1156.

Papamanoli, E., N. Tzanetakis, E. Litopoulou-Tzanetaki, and P. Kotzekidou. 2003. Characterization of lactic acid bacteria isolated 
from a Greek dry-fermented sausage in respect of their technological and probiotic properties. Meat Sci. 65:859-867.

Ramos, C. L., L. Thorsen, R. F. Schwan, and L. Jespersen. 2013. Strain-specific probiotics properties of Lactobacillus fermentum, Lactobacillus plantarum and Lactobacillus brevis isolates from Brazilian food products. Food Microbiol. 36:22-29.

Ripamonti, B., A. Agazzi, C. Bersani, P. De Dea, C. Pecorini, S. Pirani, R. Rebucci, G. Savoini, S. Stella, A. Stenico, E. Tirloni, and C. Domeneghini. 2011. Screening of species-specific lactic acid bacteria for veal calves multi-strain probiotic adjuncts. Anaerobe 17:97-105.

Rodrigues, K. L., L. R. Caputo, J. C. Carvalho, J. Evangelista, and J. M. Schneedorf. 2005. Antimicrobial and healing activity of kefir and kefiran extract. Int. J. Antimicrob. Agents 25:404-408.

Rossetti, L., and G. Giraffa. 2005. Rapid identification of dairy lactic acid bacteria by M13-generated, RAPD-PCR fingerprint databases. J. Microbiol. Methods 63:135-144.

Sánchez, B., M. Fernández-García, A. Margolles, C. G. de los ReyesGavilán, and P. Ruas-Madiedo. 2010. Technological and probiotic selection criteria of a bile-adapted Bifidobacterium animalis ssp. lactis strain. Int. Dairy J. 20:800-805.

Santos, A., M. San Mauro, A. Sanchez, J. M. Torres, and D. Marquina. 2003. The antimicrobial properties of different strains of Lactobacillus spp. isolated from Kefir. Syst. Appl. Microbiol. 26:434-437.
Servin, A. L. 2004. Antagonistic activities of lactobacilli and bifidobacteria against microbial pathogens. FEMS Microbiol. Rev. 28:405-440.

Urdaneta, E., J. Barrenetxe, P. Aranguren, A. Irigoyen, F. Marzo, and F. C. Ibáñez. 2007. Intestinal beneficial effects of kefir-supplemented diet in rats. Nutr. Res. 27:653-658.

Vinderola, G., B. Capellini, F. Villarreal, V. Suárez, A. Quiberoni, and J. Reinheimer. 2008. Usefulness of a set of simple in vitro tests for the screening and identification of probiotic candidate strains for dairy use. Lebenson. Wiss. Technol. 41:1678-1688.

Zago, M., M. E. Fornasari, D. Carminati, P. Burns, V. Suàrez, G. Vinderola, J. Reinheimer, and G. Giraffa. 2011. Characterization and probiotic potential of Lactobacillus plantarum strains isolated from cheeses. Food Microbiol. 28:1033-1040.

Zhang, Y., R. Du, L. Wang, and H. Zhang. 2010. The antioxidative effects of probiotic Lactobacillus casei Zhang on the hyperlipidemic rats. Eur. Food Res. Technol. 231:151-158.

Zheng, Y., Y. Lu, J. Wang, L. Yang, C. Pan, and Y. Huang. 2013. Probiotic properties of Lactobacillus strains isolates from Tibetan kefir grains. PLoS ONE 8:e69868. 